\title{
Relationship between Histological and Clinical Course of Psoriasis: A Pilot Investigation by Reflectance Confocal Microscopy during Goeckerman Treatment
}

\author{
Rami Archid ${ }^{a, b}$ Hans Peter Duerr ${ }^{c} \quad$ Alexa Patzelt ${ }^{a}$ Sandra Philipp ${ }^{a}$ \\ Hans-Joachim Röwert-Huber ${ }^{a}$ Martina Ulrich ${ }^{a}$ Martina Claudia Meinke ${ }^{a}$ \\ Fanny Knorra Jürgen Lademann ${ }^{a}$ \\ a Department of Dermatology, Venereology and Allergology, Charité - Universitätsmedizin Berlin, Berlin, and \\ ${ }^{b}$ Department of General and Transplant Surgery, University of Tübingen, and 'Numerus Ltd., Tübingen, Germany
}

\section{Key Words}

Psoriasis · Skin microvasculature · Capillary diameter .

Papillary diameter $\cdot$ Reflectance confocal microscopy

\begin{abstract}
Alterations of the skin microvasculature are known to play an important role in the development and maintenance of psoriatic skin lesions. In this study, we investigated lesional skin in 11 psoriatic patients during a modified Goeckerman treatment using reflectance confocal microscopy (RCM) to study the relationship between clinical clearance and histological normalization of psoriatic skin and the significance of histological abnormalities on the course of disease. The treatment regimen resulted in a significant reduction of the Psoriasis Area and Severity Index (PASI) as well as capillary and papillary diameters $(\mathrm{p}<0.0001)$. The capillary and papillary diameters were still enlarged when compared to those in normal skin $(p<0.001)$. Capillary and papillary diameters correlated with each other prior to and after treatment (correlation coefficient $=0.63$ and $0.64, p=0.01$ and 0.002 , re-
\end{abstract}

spectively) but not with the PASI. Capillary and papillary diameters after treatment and percentage reduction of the PASI during treatment seemed to be better predictors for the clinical course of relapse than the PASI after treatment. These findings make the subclinical changes of psoriatic skin vessels and dermal papillae a legitimate target for treatment. Further investigations of a large group of patients are needed to evaluate the potential of RCM findings as successor of the PASI in the monitoring of psoriasis.

(c) 2016 S. Karger AG, Basel

\section{Introduction}

Psoriasis is a chronic inflammatory skin disease with a prevalence of $0.6-4.8 \%$ in the world population [1]. Chronicity, the recurring course of psoriasis, the absence of a cure and several side effects of available treatment modalities result in a major reduction in the quality of life and the physical and psychological functioning of psoriatic patients [2-7].

\section{KARGER}

E-Mail karger@karger.com www.karger.com/spp
(C) 2016 S. Karger AG, Base

$1660-5527 / 16 / 0291-0047 \$ 39.50 / 0$
Dr. Alexa Patzelt

Center of Experimental and Applied Cutaneous Physiology, Department of Dermatology, Venereology and Allergology, Charité - Universitätsmedizin Berlin Charitéplatz 1, DE-10117 Berlin (Germany)

E-Mail alexa.patzelt@charite.de 
Although the pathomechanism of psoriasis is not yet completely understood, several findings indicate an essential role of the alteration in the skin microvasculature in the development and maintenance of psoriatic lesions [8]. The capillary loops in the psoriatic skin are elongated, widened and tortuous, especially in the papillary dermis. In contrast to those of normal skin, they largely consist of venous capillaries, characterized by the multilaminated basement membrane and bridged fenestrations of the endothelial tube [9-11]. These changes facilitate inflammatory cell migration and the nutrition of hyperplastic lesional skin $[8,9,12,13]$. Initial microvascular changes precede any psoriatic manifestation on the skin surface and occur before hyperplasia of the epidermis can be histologically detected [14-17]. The clearance of a psoriatic lesion during therapy is accompanied by a normalization of the microvascular changes, depending on the modality of therapy $[8,9,18,19]$. More recently, a clinical improvement and in some cases the healing of psoriatic skin lesions have been achieved by means of treatment with the vascular selective pulsed dye laser (PDL) $[20,21]$. However, the PDL has no effect on the inflammatory and proliferative process in psoriatic skin and induces lesion resolution by its effect on the superficial microvessels of the papillary dermis [22-24]. In a systematic review of the literature, the PDL was recommended as an effective and safe treatment option for localized plaque psoriasis [25].

Activated basal keratinocytes surrounding the dermal papillae are responsible for an elevated pro-angiogenic activity within psoriatic skin, resulting in vessel transformation in psoriasis [8]. Keratinocytes are the major source of pro-angiogenic molecules, such as the transforming growth factor- $\alpha$ [26], interleukin-8 [27], plateletderived endothelial growth factor [28] and vascular endothelial growth factor/vascular permeability factor, which is the major angiogenic factor in psoriasis $[8,29,30]$. These facts are indicative for an essential interaction between the keratinocytes and the skin microvasculature in the development and maintenance of psoriatic skin lesions.

A standardized assessment of psoriasis severity and outcome is elementary for evidence-based therapeutic management. While no biomarkers are available for the monitoring of psoriasis, a recent analysis identified 53 separate clinical measures of psoriasis severity and outcome [31], none of which meets all the validation criteria required for an ideal clinical score [31-34]. The Psoriasis Area and Severity Index (PASI) [35] represents the most common score for the assessment of psoriasis severity in clinical trials and practice. The accuracy of the PASI as a global score for psoriasis severity and for making therapeutic decisions is more than questionable $[31-33,36]$. On the other hand, reflectance confocal microscopy (RCM), a novel non-invasive, in vivo imaging technique, has been used increasingly in recent years for investigating psoriatic skin lesions, which showed a good correlation with histological findings [37-39]. In a previous study, using RCM [40] we were able to perform precise measurements of skin capillaries and papillae in normal and psoriatic skin.

According to our knowledge, to date, studies quantifying the dynamics of histological changes during Goeckerman therapy have not been performed using RCM. Furthermore, the role of subclinical structural changes of psoriatic skin at the end of treatment in the course of disease has not been investigated using non-invasive imaging techniques. Therefore, the lesional psoriatic skin during a modified Goeckerman therapy regimen was investigated in this study. Principal goals of the current study were as follows: (1) to quantify the structural abnormalities of the capillaries and papillae in psoriatic skin and their dynamics during therapy, (2) to investigate the relationship between clinical clearance and normalization of the micro-architecture of the skin and (3) to investigate the role of the status of skin papillae and capillaries at the end of therapy on the recurring course of psoriasis.

\section{Materials and Methods}

\section{Study Participants and Study Design}

The investigations were carried out on 11 patients, who had a long-standing history of chronic plaque psoriasis and were suffering from an acute relapse. Five of the patients were female and six were male (median age 51 years, mean 47.8 years). All study participants had skin phototype I-III and were healthy and normotensive. All 11 patients underwent a modified Goeckerman therapy regimen for 3-8 weeks on an outpatient basis at the Department of Dermatology, Charité - Universitätsmedizin Berlin, Germany. The study had been approved by the Ethics Committee of the Charité. We conformed to the Declaration of Helsinki as a statement of ethical principles for medical research involving human subjects. Written informed consent had been given. None of these patients had undergone systemic or local treatment (except for a keratolytic therapy with salicylates for better optical penetration) for at least 2 months prior to the investigations.

The PASI had been used for monitoring the clinical improvement and was recorded in all patients on the first and last days of therapy by the same practitioner. To monitor the structural abnormalities of the capillaries and papillae in psoriatic skin and their dynamics during therapy, a psoriatic plaque on the lower leg and/ or forearm of each participant was investigated using RCM (VivaScope ${ }^{\circledR} 1500$, Lucid Inc., Rochester, N.Y., USA), prior to therapy and on the last day of therapy.
Archid et al. 
The study participants had received instructions on the selfassessment of their disease severity on the basis of the Self-Administered PASI [41, 42]. The patients were interviewed via phone calls and followed up every 8 weeks. A Self-Administered PASI increase of $50 \%$, as compared to the PASI after therapy or the restart of an intensive psoriasis therapy regimen, was categorized as a recurrence of disease.

\section{Goeckerman Treatment Regimen}

All 11 patients received a modified Goeckerman treatment on a daily basis and 5 days a week at the Psoriasis Centre at the Department of Dermatology, Charité - Universitätsmedizin Berlin, Germany. Treatment consisted primarily of all-day UVB phototherapy in combination with $5-10 \%$ brine baths, the topical application of up to $4 \%$ anthralin (for 1-2 h/day) and crude coal tar (5-8\% liquor carbonis detergens for $4 \mathrm{~h} /$ day), with or without the application of up to $5 \%$ salicylic acid and up to $8 \%$ castor oil.

Depending on the patient's skin phototype, UVB phototherapy was started before the placement of the tar at between 20 and $40 \mathrm{~mJ} / \mathrm{cm}^{2}$ and increased daily up to an intensity of $500-1,500 \mathrm{~mJ} /$ $\mathrm{cm}^{2}$ if no burn was experienced from the previous irradiation.

Crude coal tar was used initially at a $2 \%$ concentration and anthralin at a $0.25 \%$ concentration. If the therapy had been tolerated with minimal skin irritation, the concentration was progressively increased. Salicylic acid was used in the case of induration of the lesional skin.

\section{Reflectance Confocal Microscopy}

A reflectance confocal microscope (VivaScope ${ }^{\circledR}$ 1500, Lucid Inc.) was used for the examination of the lesional psoriatic skin in this study. The VivaScope system allows using a laser beam in the near-infrared range $(830 \mathrm{~nm})$ to visualize horizontal (en face) sections of the human skin non-invasively and in vivo. Cellular details can be visualized at high resolution and good contrast in vivo without necessitating a biopsy and further histological processing or staining. Contrasts in RCM images are mainly due to variations in refractive indices of tissue structures. This technique is described in detail elsewhere [40, 43].

Confocal imaging was performed prior to therapy and on the last day of therapy according to a standardized protocol. The patients were investigated at room temperature $\left(20-24^{\circ} \mathrm{C}\right)$ and after a resting period of at least $5 \mathrm{~min}$. A psoriatic skin lesion for imaging was selected on the lower leg and/or forearm. Single confocal images of horizontal (en face) sections of the lesional skin $(500 \times$ $500 \mu \mathrm{m}$ ) were obtained from the centre of the lesion from different skin levels starting at the stratum corneum down to the papillary dermis, and partially the upper reticular dermis, at 5- $\mu \mathrm{m}$ intervals. The laser intensity was adjusted manually to achieve images with a good contrast of the dermal structures. At least 5 images of the capillary loops were taken from each participant at the level of the upper papillary dermis by scanning horizontally, in parallel to the surface of the skin.

For RCM image analysis, commercially available software (AutoCAD $2010^{\circledR}$, Autodesk Inc., San Rafael, Calif., USA) was used, which allowed precise measurements of the skin structures after calibration of the images. The Feret diameters of all clearly identified loop capillaries and dermal papillae were measured. Important principles were discussed, in order to avoid several sources of error that had occurred when this technique had been used for analysing skin components in an earlier study [40].

RCM Monitoring of Psoriasis during Goeckerman Treatment

\section{Statistical Analysis}

Due to the skewed distribution of the data of the PASI, papillary and capillary diameters, logarithmic transformations were calculated to normalize their distribution. For simplification, delogarithmized values are presented as means \pm SD. Results before and after treatment, as well as results after treatment and values of normal skin measured in an earlier study, were compared using the one-sample t test.

Correlation analysis of log-transformed measurements of the PASI, capillary and papillary diameters before and after treatment was performed. Rank correlation matrices of percentage reduction of PASI capillary and papillary diameter values were also performed. Percentage reductions were calculated as $100 \times$ (value before treatment - value after treatment)/value before treatment.

Logistic regression analysis was performed for predicting the probability of clinical relapse based on the PASI, the capillary and papillary diameters at the end of treatment as well as the percentage reduction of the PASI during therapy.

\section{Results}

\section{Clinical Response}

After a treatment lasting 19-55 days (mean 30, median 26 days), a $30-90.4 \%$ reduction of the PASI, from $19.3 \pm$ 8.2 before to $6.3 \pm 2.8$ after treatment, was achieved $(\mathrm{p}<$ 0.0001 ; table 1).

\section{Development of the Capillary Loops and Dermal}

Papillae during Therapy

During therapy, a $17-53.7 \%$ reduction of the Feret capillary diameter from $24.5 \pm 2.4 \mu \mathrm{m}$ before to $17.3 \pm 2.3$ after treatment $(\mathrm{p}<0.0001)$ and a $13.1-44.2 \%$ reduction of the Feret papillary diameter from $142.7 \pm 28.2 \mu \mathrm{m}$ before to $103.2 \pm 28.2 \mu \mathrm{m}$ after treatment $(\mathrm{p}<0.0001)$ were achieved (table 1).

The diameters of the skin microvasculature and of the dermal papillae at the end of therapy were still significantly increased when compared to the values of capillary diameter $(9.5 \pm 1.8 \mu \mathrm{m}, \mathrm{p}<0.0001)$ and papillary diameter $(69.4$ $\pm 17.1 \mu \mathrm{m}, \mathrm{p}=0.0009$ ) measured in normal skin [40] (fig. 1).

\section{Relationship between PASI, Papillary and Capillary Diameters}

Correlation analysis of log-transformed measurements of the PASI, capillary and papillary diameters before and after treatment showed that the PASI was to a high extent independent of the capillary and papillary diameters, before and after treatment $(\mathrm{r}=-0.16$ before treatment, $r=0.52$ after treatment). On the other hand, capillary and papillary diameters correlated with each other, before and after treatment (correlation coefficient $\mathrm{r}=0.63, \mathrm{p}=0.014, \mathrm{r}=0.64, \mathrm{p}=0.002$, respectively). 


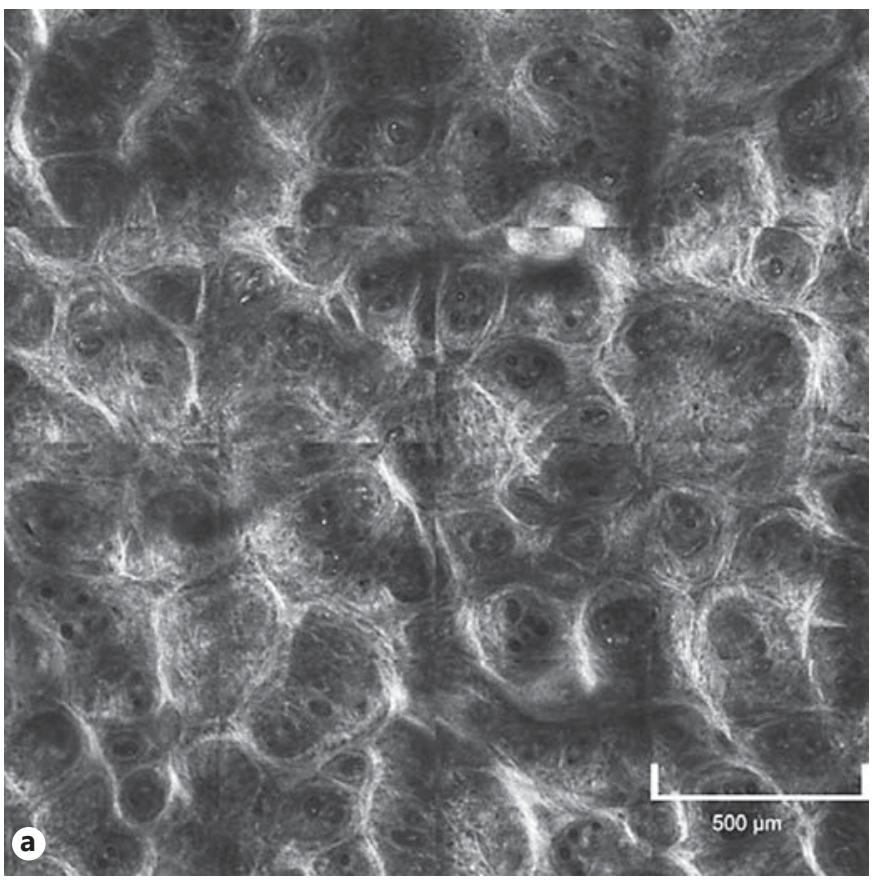

Fig. 1. Confocal image scan of a horizontal (en face) area $(2 \times$ $2 \mathrm{~mm}$ ) of the dermal-epidermal junction area in psoriatic skin prior to (a) and after (b) treatment. Following a Goeckerman therapy

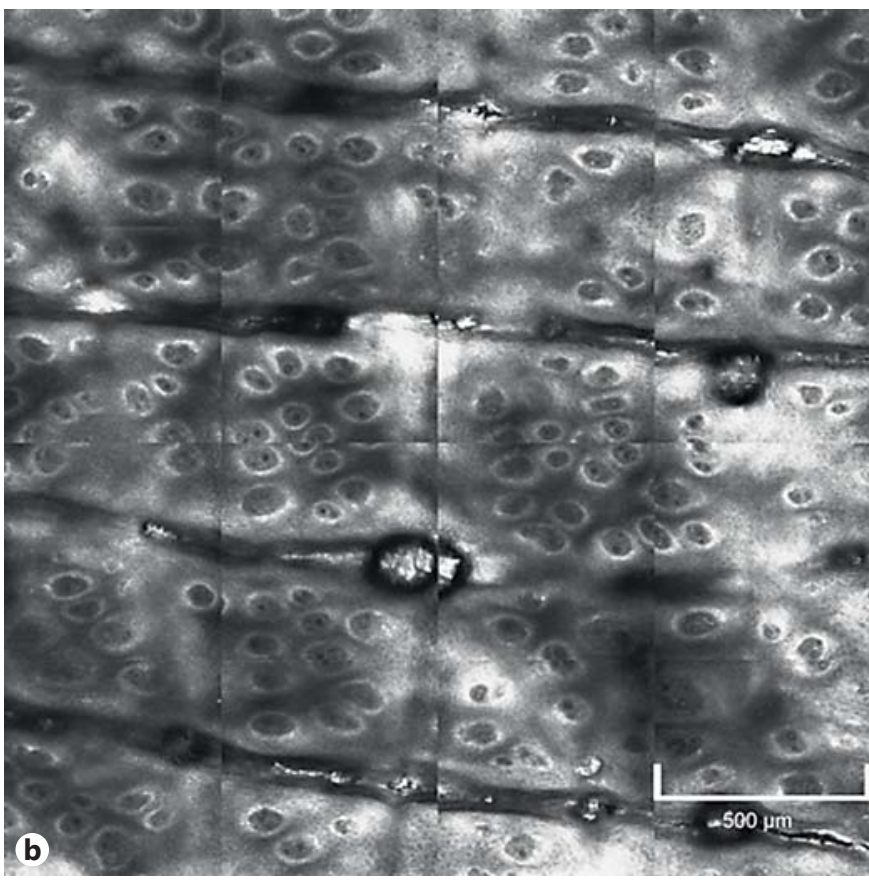

regimen, the diameters of dermal papillae and capillaries decreased during therapy but were still increased/enlarged when compared to normal skin.

Table 1. PASI, capillary $(\mathrm{C})$ and papillary $(\mathrm{P})$ diameters $(\mu \mathrm{m})$ prior to (pre) and after (post) treatment and their percentage reduction during treatment (\%red.)

\begin{tabular}{|c|c|c|c|c|c|c|c|c|c|}
\hline Patient No. & PASI pre & PASI post & \%red. PASI & C pre & C post & \%red. C & P pre & $\mathrm{P}$ post & \%red. P \\
\hline 1 & 21 & 2 & 90.4 & 27.2 & 16.0 & 41.0 & 156.3 & 72.3 & 53.7 \\
\hline 2 & 10 & 7 & 30 & 25.8 & 19.4 & 24.8 & 193.9 & 142.3 & 26.5 \\
\hline 3 & 14 & 5 & 64.2 & 26.4 & 20.2 & 23.5 & 132.1 & 106.1 & 19.7 \\
\hline 4 & 19.1 & 6.2 & 67.5 & 25.1 & 17.2 & 31.3 & 126.2 & 104 & 17.5 \\
\hline 5 & 19 & 12 & 36.8 & 26.2 & 18.8 & 27.9 & 192.7 & 165.4 & 14.1 \\
\hline 6 & 15 & 4 & 73.3 & 19.4 & 16.8 & 13.1 & 114.3 & 86.3 & 24.4 \\
\hline 7 & 16 & 6 & 62.5 & 26.9 & 20.9 & 22.4 & 150.7 & 107.4 & 28.6 \\
\hline 8 & 10 & 4 & 60 & 24.2 & 17.7 & 26.7 & 118.1 & 98 & 17 \\
\hline 9 & 40 & 10 & 75 & 24 & 13.4 & 44.2 & 136.2 & 92.7 & 31.9 \\
\hline 10 & 21 & 6 & 71.4 & 22 & 16.1 & 26.6 & 131.2 & 92.2 & 29.7 \\
\hline 11 & 25 & 8 & 68 & 22.4 & 14.2 & 36.3 & 117.5 & 68.6 & 41.5 \\
\hline
\end{tabular}

\section{Relationship between Clinical Clearance and}

Normalization of RCM Findings

A rank correlation analysis of percentage reductions in PASI, capillary and papillary diameters during treatment showed a better correlation between clinical clearance and normalization of the papillary structure $(\mathrm{r}=0.63, \mathrm{p}=$ $0.03)$ than between clinical clearance and normalization of the skin capillaries $(\mathrm{r}=0.29, \mathrm{p}=0.25$; fig. 2 ).
Relationship between Skin Status after Treatment and Recurrence

Logistic regression analysis was used to investigate the relationship between the PASI, capillary and papillary diameters after treatment and percentage reduction in the PASI on the one hand and the clinical course of the disease after treatment on the other.
50

Skin Pharmacol Physiol 2016;29:47-54 DOI: $10.1159 / 000443211$
Archid et al. 
Fig. 2. Correlation matrix of percentage reduction of capillaries (\%red. mean C), papillaries (\%red. mean P) and PASI (\%red. PASI) values. Percentage reduction is calculated as $100^{*}$ (value before - value after)/ value before. Distributions are represented in the main diagonal and correlation coefficients in corresponding graphs. Data is represented together with a $95 \%$ density ellipse and a least squares regression line with a $95 \%$ confidence region.

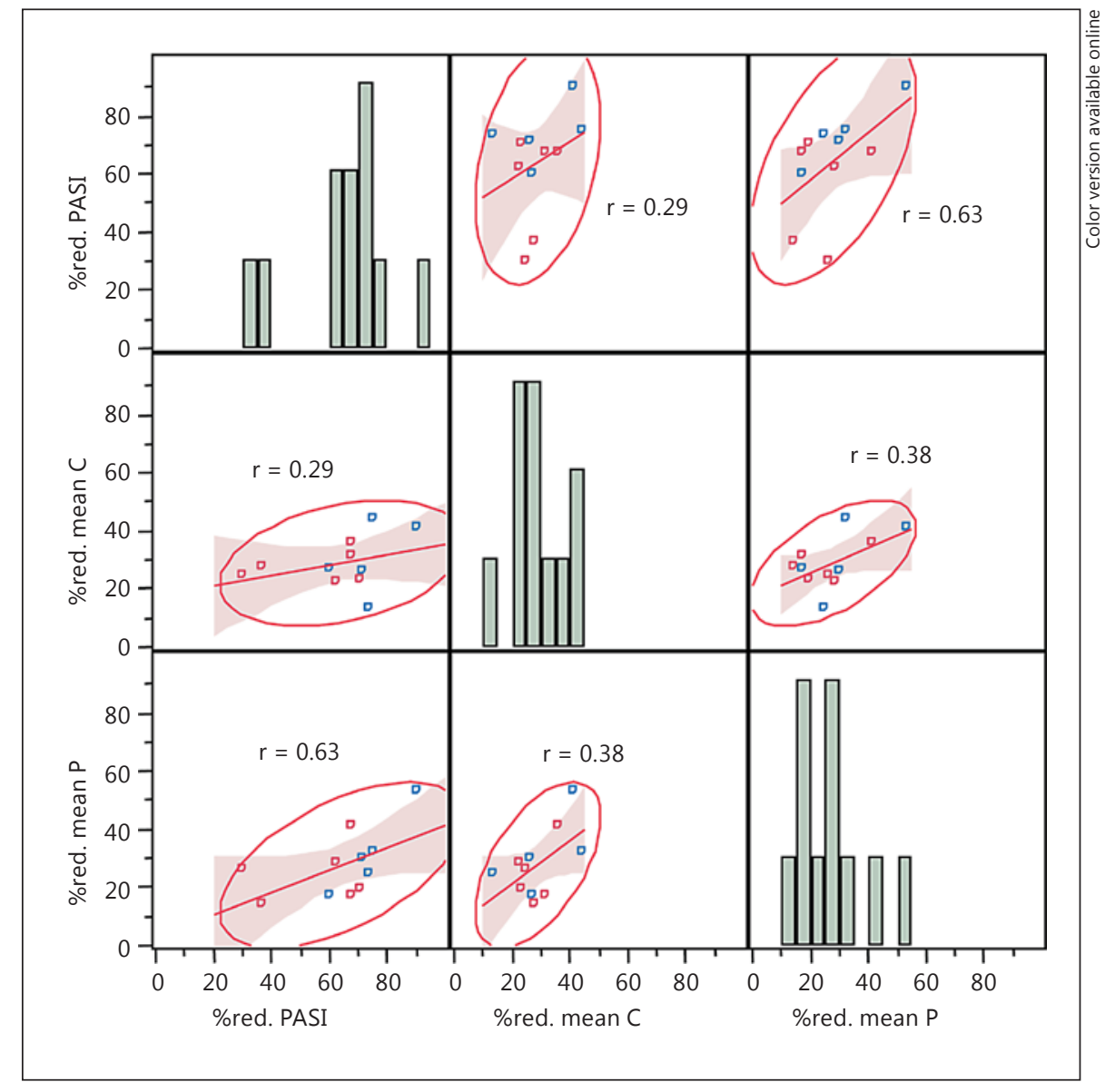

Interestingly, the analysis showed a better predictability of a psoriasis recurrence within 1 year after treatment, based on the capillary and papillary diameters after treatment ( 1 false-positive and 1 false-negative for each value) than the PASI after treatment (2 false-positives and 1 false-negative). However, the percentage reduction in PASI in this analysis showed a similar status as a predictor for recurrence, such as capillary and papillary diameters after treatment (fig. 3). Due to the small number of study participants, the logistic curves cannot be demonstrated with statistical significance $(\mathrm{p}=0.12-0.22)$.

\section{Discussion}

Findings of in vivo examination of psoriatic skin by several authors supported the hypothesis of an essential role of microvascular alteration in the development and maintenance of psoriatic skin lesions [23, 44, 45]. In a previous study, we performed exact measurements of

RCM Monitoring of Psoriasis during Goeckerman Treatment dermal capillary loops and dermal papillae using RCM [40].

In this study, the clinical improvement was associated with a significant reduction in the Feret diameters of skin capillaries and dermal papillae. Clinical improvement (percentage reduction of PASI values) correlated more with the normalization of the papillary structure $(r=0.63$, $p=0.03$ ) and correlated less with the normalization of the skin capillaries $(r=0.29, p=0.25)$. A previous study showed a significant correlation between clinical improvement of psoriasis and the reduction of the microvessel area per unit area of plaque skin, during treatment with the vessel selective PDL [23]. These differences may be attributed to the different modalities of therapy. While the Goeckerman therapy regimen has diverse anti-inflammatory and antiproliferative mechanisms of action, the effect of PDL is confined to the superficial capillary loops [22].

When logistic regression analysis was performed, the capillary and papillary diameters after treatment seemed 


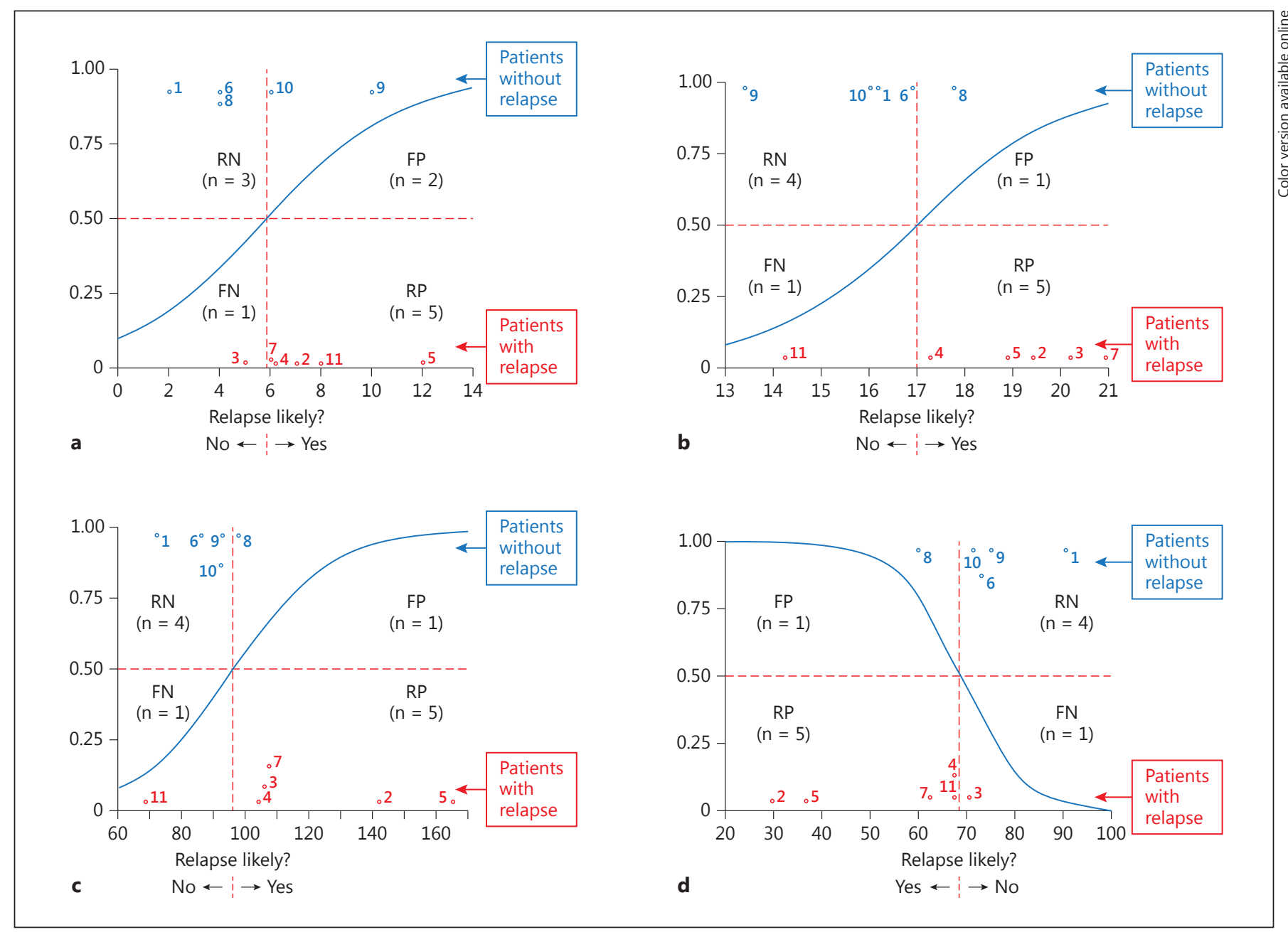

Fig. 3. Logistic regressions predicting the probability of relapse dependent on PASI after treatment (a), capillary diameter after treatment (b), papillary diameter after treatment (c) and PASI percentage reduction during treatment $(\mathbf{d})$. Patient observations are shown in horizontal direction at their value of the independent variable and in vertical direction dependent on whether they had a relapse (at the lower margin in red; colours in the online version only) or not (at the upper margin in blue). Dependent on the threshold probability for making the diagnostic decision, the graph partitions diagnostic decisions into the 4 groups of rightpositive $(\mathrm{RP})$, false-positive $(\mathrm{FP})$, right-negative $(\mathrm{RN})$, and falsenegative (FN) diagnoses. The crosshair illustrates this partitioning for a threshold probability of relapse. to be a better predictor for the clinical course of psoriasis than the PASI. Due to the low number of study participants, the logistic regression analysis was not statistically significant $(\mathrm{p}=0.12-0.22)$. Many factors, e.g. smoking, alcohol consumption, diet, infections, drugs and stressful life events, play a role in furthering a recurrence of psoriasis [1]. These factors may manipulate the results to a higher extent in the case of a low number of patients. However, the results of this analysis let us assume at least a better tendency for capillary and papillary diameters in predicting the course of psoriasis after treatment and gen- erally in measuring the effectiveness of therapies when compared to the PASI. The percentile reduction in the PASI showed a similar status as a predictor for recurrence like capillary and papillary diameters after treatment. However, the percentage reduction in the PASI depends on the clinical skin status before and after treatment and does not reflect the impairment of the skin after treatment.

Capillary and papillary diameters correlated with each other before and after treatment. This correlation supports the assumption of a relationship between the alter- 
ation of the skin vessels and the activation of basal keratinocytes. Although the widespread use of the PASI simplifies the comparison of results among clinical trials, the PASI has frequently been criticized for its questionable validity, low responsiveness and reliability $[31,33]$. Especially in the most relevant range (PASI 0-20), the PASI shows a low interrater agreement, which results in a weak concordance in the decision to treat [33]. In this study, the PASI was to a high extent independent of the capillary and papillary diameters, before and after treatment. However, valid measures for psoriasis severity and outcome are indispensable for the assessment of psoriasis in clinical trials and for evidence-based decisions in clinical practice.

RCM represents a promising non-invasive technique for evaluating structural changes in psoriatic skin in vivo and for monitoring the therapeutic response on a microscopic level. RCM investigation does not interfere with the different topical treatment modalities used for psoriasis. Side effects of antipsoriatic therapies and costs are limiting factors for the duration of treatment. The changes in the dermal papillae and superficial microvessels of psoriatic skin represent a legitimate target for antipsoriatic therapy, and their clearance status should be taken into consideration, in order to determine the duration of therapy. A complete or nearly complete clearance of these alterations may be helpful in extending the effect of therapy and prolonging the period to the next recurrence of disease. Parameters of dermal papillae and microvessels obtained non-invasively are likely to be good candidates for a better standardization of outcome in clinical practice and trials. Their potential as a successor of the PASI should be evaluated in studies with a higher number of patients, whereby routine usage could improve our understanding of psoriasis and the effectiveness of treatment.

\section{Acknowledgements}

We would like to thank the Foundation 'Skin Physiology' of the Donor Association for German Science and Humanities for financial support.

\section{Disclosure Statement}

The authors state no conflict of interest.

\section{References}

1 Naldi L: Epidemiology of psoriasis. Curr Drug Targets Inflamm Allergy 2004;3:121128.

2 Langley RG, Krueger GG, Griffiths CE: Psoriasis: epidemiology, clinical features, and quality of life. Ann Rheum Dis 2005;64(suppl 2):ii18-ii23; discussion ii24-ii25.

3 Sampogna F, Sera F, Abeni D: Measures of clinical severity, quality of life, and psychological distress in patients with psoriasis: a cluster analysis. J Invest Dermatol 2004;122: 602-607.

4 De Korte J, Sprangers MA, Mombers FM, Bos JD: Quality of life in patients with psoriasis: a systematic literature review. J Invest Dermatol Symp Proc 2004;9:140-147.

5 Rapp SR, Feldman SR, Exum ML, Fleischer $\mathrm{AB}$ Jr, Reboussin DM: Psoriasis causes as much disability as other major medical diseases. J Am Acad Dermatol 1999;41:401-407.

6 Krueger G, Koo J, Lebwohl M, Menter A, Stern RS, Rolstad T: The impact of psoriasis on quality of life: results of a 1998 National Psoriasis Foundation patient-membership survey. Arch Dermatol 2001;137:280-284.

7 Farkas A, Kemeny L: Alcohol, liver, systemic inflammation and skin: a focus on patients with psoriasis. Skin Pharmacol Physiol 2013; 26:119-126.
8 Micali G, Lacarrubba F, Musumeci ML, Massimino D, Nasca MR: Cutaneous vascular patterns in psoriasis. Int J Dermatol 2010;49: 249-256.

9 Braverman IM, Yen A: Ultrastructure of the capillary loops in the dermal papillae of psoriasis. J Invest Dermatol 1977;68:53-60.

10 Braverman IM: Electron microscopic studies of the microcirculation in psoriasis. J Invest Dermatol 1972;59:91-98.

11 Braverman IM, Yen A: Microcirculation in psoriatic skin. J Invest Dermatol 1974;62: 493-502.

12 Braverman IM: The cutaneous microcirculation. J Invest Dermatol Symp Proc 2000;5:39.

13 Krueger G, Ellis CN: Psoriasis - recent advances in understanding its pathogenesis and treatment. J Am Acad Dermatol 2005;53:S94S100.

14 Pinkus H, Mehregan AH: The primary histologic lesion of seborrheic dermatitis and psoriasis. J Invest Dermatol 1966;46:109-116.

15 Speight EL, Essex TJ, Farr PM: The study of plaques of psoriasis using a scanning laserDoppler velocimeter. Br J Dermatol 1993; 128: 519-524.
16 Goodfield M, Hull SM, Holland D, Roberts G, Wood E, Reid S, Cunliffe W: Investigations of the 'active' edge of plaque psoriasis: vascular proliferation precedes changes in epidermal keratin. Br J Dermatol 1994;131:808-813.

17 Navarro R, Dauden E, Gallo E, Sanchez-Mateos DS, Garcia-Diez A: Alopecia areata during treatment of psoriasis with adalimumab and leflunomide: a case and review of the literature. Skin Pharmacol Physiol 2012;25:107-110.

18 Gordon M, Johnson WC, Burgoon CF Jr: Histopathology and histochemistry of psoriasis. II. Dynamics of lesions during treatment. Arch Pathol 1967;84:443-450.

19 Braverman IM, Sibley J: The response of psoriatic epidermis and microvessels to treatment with topical steroids and oral methotrexate. J Invest Dermatol 1985;85:584-586.

20 Hacker SM, Rasmussen JE: The effect of flash lamp-pulsed dye laser on psoriasis. Arch Dermatol 1992;128:853-855.

21 Katugampola GA, Rees AM, Lanigan SW: Laser treatment of psoriasis. Br J Dermatol 1995; 133:909-913.

22 Hern S, Allen MH, Sousa AR, Harland CC, Barker JN, Levick JR, Mortimer PS: Immunohistochemical evaluation of psoriatic plaques following selective photothermolysis of the superficial capillaries. Br J Dermatol 2001; 145:45-53. 
23 Hern S, Stanton AW, Mellor RH, Harland CC, Levick JR, Mortimer PS: In vivo quantification of the structural abnormalities in psoriatic microvessels before and after pulsed dye laser treatment. Br J Dermatol 2005; 152:505511.

24 Korting HC, Schollmann C, Willers C, Wigger-Alberti W: Bioavailability, antipsoriatic efficacy and tolerability of a new light cream with mometasone furoate $0.1 \%$. Skin Pharmacol Physiol 2012;25:133-141.

25 Erceg A, de Jong EM, van de Kerkhof PC, Seyger MM: The efficacy of pulsed dye laser treatment for inflammatory skin diseases: a systematic review. J Am Acad Dermatol 2013; 69:609-615.

26 Elder JT, Fisher GJ, Lindquist PB, Bennett GL, Pittelkow MR, Coffey RJ Jr, Ellingsworth L, Derynck R, Voorhees JJ: Overexpression of transforming growth factor alpha in psoriatic epidermis. Science 1989;243:811-814.

27 Nickoloff BJ, Mitra RS, Varani J, Dixit VM, Polverini PJ: Aberrant production of interleukin- 8 and thrombospondin-1 by psoriatic keratinocytes mediates angiogenesis. Am J Pathol 1994;144:820-828.

28 Creamer D, Jaggar R, Allen M, Bicknell R, Barker J: Overexpression of the angiogenic factor platelet-derived endothelial cell growth factor/thymidine phosphorylase in psoriatic epidermis. Br J Dermatol 1997; 137: 851-855.

29 Detmar M, Brown LF, Claffey KP, Yeo KT, Kocher O, Jackman RW, Berse B, Dvorak HF: Overexpression of vascular permeability factor/vascular endothelial growth factor and its receptors in psoriasis. J Exp Med 1994;180: 1141-1146.

30 Detmar M, Yeo KT, Nagy JA, Van de Water L, Brown LF, Berse B, Elicker BM, Ledbetter
S, Dvorak HF: Keratinocyte-derived vascular permeability factor (vascular endothelial growth factor) is a potent mitogen for dermal microvascular endothelial cells. J Invest Dermatol 1995; 105:44-50.

31 Spuls PI, Lecluse LL, Poulsen ML, Bos JD, Stern RS, Nijsten T: How good are clinical severity and outcome measures for psoriasis? Quantitative evaluation in a systematic review. J Invest Dermatol 2010;130:933-943.

32 Ashcroft DM, Wan Po AL, Williams HC, Griffiths CE: Clinical measures of disease severity and outcome in psoriasis: a critical appraisal of their quality. Br J Dermatol 1999; 141:185-191.

33 Gourraud PA, Le Gall C, Puzenat E, Aubin F, Ortonne JP, Paul CF: Why statistics matter: limited inter-rater agreement prevents using the Psoriasis Area and Severity Index as a unique determinant of therapeutic decision in psoriasis. J Invest Dermatol 2012;132:21712175.

34 Ramadan R, Tawdy A, Abdel Hay R, Rashed L, Tawfik D: The antioxidant role of paraoxonase 1 and vitamin $\mathrm{E}$ in three autoimmune diseases. Skin Pharmacol Physiol 2013;26:7.

35 Fredriksson T: Oral treatment of psoriasis and pustulosis palmo-plantaris with Ro 109359. Dermatologica 1978;157(suppl 1):1318.

36 Pazyar N, Yaghoobi R: Tea tree oil as a novel antipsoriasis weapon. Skin Pharmacol Physiol 2012;25:162-163.

37 Koller S, Gerger A, Ahlgrimm-Siess V, Weger W, Smolle J, Hofmann-Wellenhof R: In vivo reflectance confocal microscopy of erythematosquamous skin diseases. Exp Dermatol 2009; 18:536-540.

38 Ardigo M, Cota C, Berardesca E, Gonzalez S: Concordance between in vivo reflectance confocal microscopy and histology in the evaluation of plaque psoriasis. J Eur Acad Dermatol Venereol 2009;23:660-667.

39 Wolberink EA, van Erp PE, de Boer-van Huizen RT, van de Kerkhof PC, Gerritsen MJ: Reflectance confocal microscopy: an effective tool for monitoring ultraviolet $\mathrm{B}$ phototherapy in psoriasis. Br J Dermatol 2012;167:396403.

40 Archid R, Patzelt A, Lange-Asschenfeldt B, Ahmad SS, Ulrich M, Stockfleth E, Philipp S, Sterry W, Lademann J: Confocal laser-scanning microscopy of capillaries in normal and psoriatic skin. J Biomed Opt 2012;17:101511.

41 Fleischer AB Jr, Rapp SR, Reboussin DM, Vanarthos JC, Feldman SR: Patient measurement of psoriasis disease severity with a structured instrument. J Invest Dermatol 1994; 102:967-969.

42 Fleischer AB Jr, Feldman SR, Rapp SR, Reboussin DM, Exum ML, Clark AR, Rajashekhar V: Disease severity measures in a population of psoriasis patients: the symptoms of psoriasis correlate with self-administered Psoriasis Area and Severity Index scores. J Invest Dermatol 1996;107:26-29.

43 Rajadhyaksha M, Gonzalez S, Zavislan JM, Anderson RR, Webb RH: In vivo confocal scanning laser microscopy of human skin. II. Advances in instrumentation and comparison with histology. J Invest Dermatol 1999; 113:293-303.

44 Hern S, Mortimer PS: In vivo quantification of microvessels in clinically uninvolved psoriatic skin and in normal skin. Br J Dermatol 2007;156:1224-1229.

45 Rosina P, Giovannini A, Gisondi P, Girolomoni G: Microcirculatory modifications of psoriatic lesions during topical therapy. Skin Res Technol 2009;15:135-138. 